\title{
A Local Study on Antenatal Features of Preterm Births at 26-32 Versus 33-36 Weeks of Pregnancy
}

\author{
Fusun VAROL ${ }^{1}$, Nuray ER¹, Necdet SUT'², Cenk N. SAYIN ${ }^{1}$ \\ Edirne, Turkey
}

\section{ABSTRACT}

OBJECTIVE: The antenatal features of pregnancies affect the incidence of preterm births. This retrospective study from Trakya University of Northwestern Turkey, describes antenatal factors involved in preterm births at 26-32 weeks of pregnancy and compares with those involved in preterm births at 3336 weeks.

STUDY DESIGN: The records of preterm births at 26-32 weeks (earlier preterm births, $n=419$ ) and at 33-36 weeks (later preterm births, $n=158$ ) during the years 2002-2010 were reviewed and the demographic, obstetric and medical features were evaluated retrospectively. The data was expressed as numbers and percentages and analyzed by SPSS 20.0 .

RESULTS: Iron supplementation [OR $0.27(0.16-0.45), p=<0.001]$, short cervix [OR 9.12 (2.09-39.73) $p=0.003$ ] and infection [OR $2.6(1.2-5.6) p=0.014]$ were important factors in the emergence of earlier preterm births which occurred at the rate of $1.4 \%$.

CONCLUSIONS: Earlier preterm births at 26-32 weeks of pregnancy, which compose an obstetric issue, are associated with several antenatal risk factors such as nutrition, cervical problems and infections in Northwestern Turkey.

Keywords: Antenatal features, Earlier preterm births

\section{Gynecol Obstet Reprod Med 2018;24(1):1-6}

\section{Introduction}

The World Health Organization defines preterm birth (PTB) as birth before 37 completed weeks of gestation, regardless of fetal weight. There is a burden of 15 million PTBs occurring annually worldwide, which leads to about one million neonatal deaths $(1,2,3)$. The earlier PTBs before 33 completed weeks of gestation carry particular importance with high perinatal morbidity and mortality. Therefore, to characterize PTB associated local and even time-dependent antenatal risk factors, is of great significance in prevention. Being aware of the antenatal risk factors may lead to avoid these fac-

\footnotetext{
${ }^{1}$ Trakya University, School of Medicine, Department of Obstetrics and Gynecology, and 'Department of Bioistatistics, Edirne

Address of Correspondence: Fusun Varol

Trakya University, School of Medicine,

Department of Obstetrics and

Gynecology 22030 Edirne, Turkey

fgvarol@yahoo.com

Submitted for Publication: $\quad 24.03 .2017$

Accepted for Publication: $\quad 03.04 .2017$
}

\begin{tabular}{|c|c|}
\hline \multicolumn{2}{|c|}{ Access this article online } \\
\hline $\begin{array}{c}\text { Quick Response Code: } \\
\text { 만젼 }\end{array}$ & $\begin{array}{l}\text { Website: www.gorm.com.tr } \\
\text { e- mail: info@gorm.com.tr }\end{array}$ \\
\hline 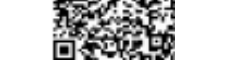 & DOI:10.201613/GORM.2016.695 \\
\hline
\end{tabular}

How to cite this article: Varol F. Er N. Sut N. Sayin CN. A Local Study on Antenatal Features of Preterm Births at 26-32 Versus 33-36 Weeks of Pregnancy. Gynecol Obstet Reprod Med 2018;24(1):1-6 tors by taking preventive measures such as smoking cessation, treating infections and others.

The objective of our study performed at Northwestern Turkey, was to define regional antenatal features of preterm births which might differ between the earlier ones at 26-32 weeks and the later ones at 33-36 weeks, and also to analyze them with respect to the type of onset of labor.

\section{Material and Method}

The PTBs which occurred at Trakya University of Northwestern Turkey between January 2002 and December 2010 , were analyzed in this study. During this time period, totally 29841 live births were encountered in the Northwestern Turkey. The study data included only the PTBs with live-born neonates and was obtained from neonatal intensive care unit (NICU) of Trakya University. Pregnancies with fetal anomalies and multiple pregnancies were excluded. The PTBs were grouped as earlier PTBs at $26^{0 / 7}-32^{6 / 7}$ weeks of pregnancy (EPTBs) and later PTBs at $33^{0 / 7}-36^{6 / 7}$ weeks of pregnancy (LPTBs). The antenatal records of EPTBs $(n=419)$ and LPTBs $(n=158)$ were evaluated retrospectively and the antenatal factors involved were also analyzed according to the type of labor onset, as spontaneous PTB (SPTB), medically indicated PTB (MIPTB) and PTB with premature rupture of the membranes (PROMPTB). The study was approved by the local ethic committee (TÜTF-GOKAEK 2014/32). 
Gestational age determinations of the PTBs based on last menstrual period correlated with ultrasound measurements. The socio-demographic data included maternal age, education, marital status, prenatal care, iron $(\mathrm{Fe})$ intake, body mass index (BMI) and smoking.

Adolescent pregnancy was defined as maternal age being $<19$ years old. Education was categorized as $\leq 8$ years and $>8$ years. Marital status was accepted as single if non-legally married. Prenatal care was considered to be regular when there were at least 5 regular visits during antenatal period. The maternal Fe intake was based on self-report. BMI was calculated by dividing the pre-pregnancy body weight by the square of height $\left(\mathrm{kg} / \mathrm{m}^{2}\right)$ and classified as underweight $(<18.5)$, normal (18.5-24.9), overweight (25-29.9) and obese ( $\geq 30)$. Smoking was also based on maternal self-report.

Hypertension was defined as blood pressure $\geq 140 / 90$ $\mathrm{mmHg}$ on two or more occasions before or during pregnancy with or without proteinuria ( $\geq 0.3 \mathrm{gr} / 24$ hours). The other medical disorders were glucose intolerance (Gestational diabetes and preexisting diabetes mellitus) and goiter with hypothyroidism. History of recurrent spontaneous abortions ( $\geq 2$ abortions) was extracted from the medical charts.

Third trimester bleeding due to sonographically diagnosed placental problems (placenta previa, placental abruption), in- fections (urinary tract infection and chorioamnionitis with Creactive protein $>12 \mathrm{mg} / \mathrm{L}$ ), PPROM (preterm premature rupture of the membranes before 37 weeks), short cervix $(<25$ $\mathrm{mm}$ ), maternal anemia (hemoglobin values $<10.5 \mathrm{gr} / \mathrm{dL}$ ) were some other clinical features recorded.

Results were expressed as numbers and percentages (\%) and compared with univariate analysis. Then odds ratios (OR) (95\% confidence interval-CI) were calculated with multivariate logistic regression. The data obtained from this study was analyzed by SPSS 20.0 (IBM SPSS Inc., Chicago, IL, USA). $\mathrm{p}<0.05$ was considered as statistically significant.

\section{Results}

The data on antenatal features related to PTBs is presented in table 1. The incidence of adolescent pregnancies was $9.1 \%$ in the group with PTB at 26-32 weeks (EPTB) and 4.4\% in the group with PTB at 33-36 weeks (LPTB) $(\mathrm{p}>0.05)$. Lower maternal educational level ( $\leq 8$ years) was significantly more common in the EPTB group (37\% vs $24.1 \%)(\mathrm{p}=0.003)$. However, the association of education with EPTBs was not significant in the multivariate analysis. The incidences of smoking were $18.6 \%$ and $20.3 \%$ in EPTB and LPTB groups respectively ( $\mathrm{p}>0.05)$. Among the EPTBs $7.4 \%$ of the mothers were single and this was significantly higher than the $1.3 \%$

Table 1: Comparison of demographic and clinical features of preterm births at 26-32 weeks and 33-36 weeks

\begin{tabular}{|c|c|c|c|c|c|c|}
\hline & \multicolumn{3}{|c|}{ Univariate analysis } & \multicolumn{3}{|c|}{ Multivariate logistic regression } \\
\hline & $\begin{array}{c}\text { EPTBs }(n=419) \\
n(\%)\end{array}$ & $\begin{array}{c}\text { LPTBs }(\mathrm{n}=158) \\
\mathrm{n}(\%)\end{array}$ & p & OR & $95 \% \mathrm{Cl}$ & $p$ \\
\hline Age & & & 0.169 & - & - & - \\
\hline $20-34$ y & $325(77.6)$ & $127(80.4)$ & & & & \\
\hline$<19$ y & $38(9.1)$ & $7(4.4)$ & & & & \\
\hline$\geq 35$ y & $56(13.4)$ & $24(15.2)$ & & & & \\
\hline Education $\leq 8$ years & $264(63.0)$ & $120(75.9)$ & 0.003 & 0.839 & $0.528-1.335$ & 0.459 \\
\hline Smoking, yes & $78(18.6)$ & $32(20.3)$ & 0.655 & & & \\
\hline Single, yes & $31(7.4)$ & $2(1.3)$ & 0.009 & 2.608 & $0.574-11.853$ & 0.214 \\
\hline$B M I$ & & & $<0.001$ & & & \\
\hline Normal (18.5-24.9) & $128(30.5)$ & $30(19.0)$ & & 1 & Reference category & \\
\hline Overweight (25-29.9) & $241(57.5)$ & $123(77.8)$ & & 0.510 & $0.313-0.829$ & 0.007 \\
\hline Obese $(\geq 30)$ & $50(11.9)$ & $5(3.2)$ & & 3.214 & $1.138-9.081$ & 0.028 \\
\hline Regular antenatal care, yes & $303(72.3)$ & $114(72.2)$ & 0.969 & - & - & - \\
\hline Recurrent abortion $\geq 2$, yes & $44(10.5)$ & $11(7.0)$ & 0.197 & - & - & - \\
\hline Previous premature birth, yes & $45(10.7)$ & $15(9.5)$ & 0.662 & - & - & - \\
\hline Short cervix, yes & $34(8.1)$ & $2(1.3)$ & 0.005 & 9.123 & $2.095-39.731$ & 0.003 \\
\hline Fe intake, yes & $241(57.5)$ & $134(84.8)$ & $<0.001$ & 0.271 & $0.163-0.451$ & $<0.001$ \\
\hline Anemia, yes & $143(34.1)$ & $41(25.9)$ & 0.060 & 1.329 & $0.844-2.091$ & 0.219 \\
\hline Infection, yes & $70(16.7)$ & $9(5.7)$ & 0.001 & 2.605 & $1.210-5.606$ & 0.014 \\
\hline Placental problems, yes & $49(11.7)$ & $7(4.4)$ & 0.013 & 2.712 & $1.153-6.379$ & 0.022 \\
\hline Hypertension in pregnancy, yes & $99(23.6)$ & $32(20.3)$ & 0.388 & 1.506 & $0.914-2.479$ & 0.108 \\
\hline Other maternal diseases, yes & $25(6.0)$ & $12(7.6)$ & 0.602 & 0.948 & $0.422-2.129$ & 0.898 \\
\hline
\end{tabular}

EPTBs: Earlier preterm births (at 26-32 weeks), LPTBs: Later preterm births (at 33-36 weeks) 
rate observed among the LPTBs $(p=0.009)$, but the impact of being single on EPTB was not significant in the multivariate analysis. Regarding BMI, the ratio of overweight mothers was significantly lower in the EPTB group (57.5\% vs $77.8 \%$ ) $(p<0.001)$, but the ratio of obese mothers was significantly higher $(11.9 \%$ vs $3.2 \%)(p<0.001)$. Taking normal BMI as reference, the OR values for the association of being overweight and obese with EPTB were $0.51(0.31-0.82 ; \mathrm{p}=0.007)$ and 3.2 (1.13-9.08 $\mathrm{p}=0.028$ respectively, appearing to be strongly significant. The rates of regular antenatal care, history of recurrent abortion and previous PTB were not significantly different between the EPTB and LPTB groups. Short cervix was observed at a significantly higher rate in the EPTB group, compared to the LPTB group $(8.1 \%$ vs $1.3 \%)(p=0.005)$. The association of short cervix with EPTB was also significant [OR $9.12(2.09-39.73) \mathrm{p}=0.003]$. Fe intake was significantly less among the EPTBs in comparison with LPTBs $(p<0.001)$ and it showed significant association with EPTBs in the multivariate analysis [OR $0.27(0.16-0.45) \mathrm{p}<0.001]$. Anemia was found in $34.1 \%$ of EPTBs. Infection was present in $16.7 \%$ of EPTBs, which was significantly higher than the $5.7 \%$ rate present in the LPTBs $(p=0.001)$. In the multivariate analysis, the association of infection with EPTB was also significant [OR 2.6 (1.21-5.60) $\mathrm{p}=0.014]$. Third trimester bleeding due to sonographically diagnosed placental problems was observed in $11.7 \%$ of EPTBs, compared to $4.4 \%$ in LPTBs, where the difference was statistically significant $(p=0.013)$. In the multivariate analysis, the association of placental problems with EPTB was significant as well [OR $2.7(1.15-6.38) \mathrm{p}=0.022$ ]. There wasn't any significant difference between EPTBs and LPTBs regarding hypertension in pregnancy $(23.6 \%$ vs $20.3 \%, \mathrm{p}>0.05)$ or other maternal disorders such as goiter and diabetes $(6 \%$ vs $7.6 \%, \mathrm{p}>0.05)$.

These antenatal features of PTBs were analyzed according to type of onset of labor in table 2. As observed, among the SPTBs and PROMPTBs, adolescent pregnancies were more common in the EPTB group (10\% and $9.7 \%$ respectively) compared to the LPTB group (3.6\% and 5.1\% respectively), although not statistically significant; whereas among the MIPTBs, $20.2 \%$ of the EPTBs had advanced maternal age ( $\geq 35$ years). Furthermore, in $32.9 \%$ of spontaneous EPTBs and

Table 2: The characteristics of preterm births at 26-32 weeks and 33-36 weeks with respect to the type of labor onset

\begin{tabular}{|c|c|c|c|c|c|c|c|c|c|}
\hline & \multicolumn{2}{|c|}{$\begin{array}{l}\text { SPTBs } \\
(n=195)\end{array}$} & \multirow[b]{2}{*}{$p$} & \multicolumn{2}{|c|}{$\begin{array}{l}\text { MIPTBs } \\
(n=158)\end{array}$} & \multirow[b]{2}{*}{$p$} & \multicolumn{2}{|c|}{$\begin{array}{l}\text { PROMPTBs } \\
(n=224)\end{array}$} & \multirow[b]{2}{*}{$p$} \\
\hline & $\begin{array}{c}\text { EPTBs } \\
(n=140) \\
n(\%)\end{array}$ & $\begin{array}{c}\text { LPTBs } \\
(n=55) \\
n(\%)\end{array}$ & & $\begin{array}{c}\text { EPTBs } \\
(n=114) \\
n(\%)\end{array}$ & $\begin{array}{c}\text { LPTBs } \\
(n=44) \\
n(\%)\end{array}$ & & $\begin{array}{c}\text { EPTBs } \\
(n=165) \\
n(\%)\end{array}$ & $\begin{array}{c}\text { LPTBs } \\
(n=59) \\
n(\%)\end{array}$ & \\
\hline Age & & & 0.291 & & & 0.796 & & & 0.503 \\
\hline $20-34$ y & $111(79.3)$ & $45(81.8)$ & & $83(72.8)$ & $34(77.3)$ & & $131(79.4)$ & $48(81.4)$ & \\
\hline$<19 y$ & $14(10.0)$ & $2(3.6)$ & & $8(7.0)$ & $2(14.2)$ & & $16(9.7)$ & $3(5.1)$ & \\
\hline$\geq 35 \mathrm{y}$ & $15(10.7)$ & $8(14.5)$ & & $23(20.2)$ & $8(18.2)$ & & $17(10.3)$ & $8(13.6)$ & \\
\hline Education & & & 0.042 & & & 0.671 & & & 0.013 \\
\hline$\leq 8$ years & 46 (32.9) & $10(18.2)$ & & $43(37.7)$ & $15(34.1)$ & & $66(40.0)$ & $13(22.0)$ & \\
\hline$>8$ years & $94(67.1)$ & $45(81.8)$ & & $71(62.3)$ & $29(65.9)$ & & $99(60.0)$ & $46(78.0)$ & \\
\hline Smoking, yes & $23(16.4)$ & $12(21.8)$ & 0.377 & $11(9.6)$ & $6(13.6)$ & 0.468 & $44(26.7)$ & $14(23.7)$ & 0.658 \\
\hline Single, yes & $9(6.4)$ & $1(1.8)$ & 0.287 & $6(5.3)$ & $0(0.0)$ & 0.187 & $16(9.7)$ & $1(1.7)$ & 0.048 \\
\hline BMI & & & 0.001 & & & 0.117 & & & 0.043 \\
\hline Normal (18.5-24.9) & $43(30.7)$ & $8(14.5)$ & & $28(24.6)$ & $8(18.2)$ & & $57(34.5)$ & $14(23.7)$ & \\
\hline Overweight (25-29.9) & $76(54.3)$ & $45(81.8)$ & & $70(61.4)$ & $34(77.3)$ & & $95(57.6)$ & $44(74.6)$ & \\
\hline Obese $(\geq 30)$ & $21(15)$ & $2(3.6)$ & & $16(14)$ & $2(4.5)$ & & $13(7.9)$ & $1(1.7)$ & \\
\hline Regular antenatal care, yes & $113(80.7)$ & $44(80.0)$ & 1.000 & $86(75.4)$ & $34(77.3)$ & 0.973 & $104(63.0)$ & $36(61.0)$ & 0.906 \\
\hline Recurrent abortion $\geq 2$, yes & $17(12.1)$ & $5(9.1)$ & 0.723 & $7(6.1)$ & $4(9.1)$ & 0.501 & $20(12.1)$ & $2(3.4)$ & 0.093 \\
\hline Previous premature birth, yes & $11(7.9)$ & $5(9.1)$ & 0.776 & $15(13.2)$ & $5(11.4)$ & 0.761 & $19(11.5)$ & $5(8.5)$ & 0.517 \\
\hline Short cervix, yes & $22(15.7)$ & $1(1.8)$ & 0.007 & $1(0.9)$ & $0(0.0)$ & 1.000 & $11(6.7)$ & $1(1.7)$ & 0.191 \\
\hline Fe intake, yes & $85(60.7)$ & $48(87.3)$ & $<0.001$ & $67(58.8)$ & $40(90.9)$ & $<0.001$ & $89(53.9)$ & $46(78.0)$ & 0.001 \\
\hline Anemia, yes & $37(26.4)$ & $14(25.5)$ & 0.889 & $36(31.6)$ & $6(13.6)$ & 0.022 & $70(42.4)$ & $21(35.6)$ & 0.359 \\
\hline Infection, yes & $14(10.0)$ & $1(1.8)$ & 0.071 & $15(13.2)$ & $3(6.8)$ & 0.261 & $41(24.8)$ & $5(8.5)$ & 0.008 \\
\hline Placental problems, yes & $6(4.3)$ & $4(7.3)$ & 0.395 & $30(26.3)$ & $1(2.3)$ & 0.001 & $13(7.9)$ & $2(3.4)$ & 0.364 \\
\hline Hypertension in pregnancy, yes & $13(9.3)$ & $1(1.8)$ & 0.119 & $76(66.7)$ & $29(65.9)$ & 1.000 & $10(6.1)$ & $2(3.4)$ & 0.737 \\
\hline Other maternal diseases, yes & $10(7.1)$ & $1(1.8)$ & 0.186 & $7(6.1)$ & $9(20.5)$ & 0.015 & $8(4.8)$ & $2(3.4)$ & 1.000 \\
\hline
\end{tabular}

SPTBs: Spontaneous preterm births, MIPTBs: Medically indicated preterm births, PROMPTB: Preterm birth with premature rupture of the membranes, EPTBs: Earlier preterm births (at 26-32 weeks), LPTBs: Later preterm births (at 33-36 weeks) 
$40 \%$ of EPTBs with PROM, maternal education was $\leq 8$ years. These were significantly different from the rates of $18.2 \%$ and $22 \%$ recorded for the LPTB group with spontaneous onset of labor and PROM respectively. Although not statistically significant, smoking was less frequent among the EPTBs compared to the LPTBs within the MIPTB group $(9.6 \%$ versus $13.6 \%$ respectively, $\mathrm{p}>0.05$ ). On the other hand, being single was more frequent among the EPTBs compared to the LPTBs within the PROMPTB group (9.7\% versus $1.7 \%$ respectively), which was statistically significant $(\mathrm{p}=0.048)$. The rate of regular antenatal care was not significantly different between the EPTBs and LPTBs within any of the three groups with different types of labor onset. Short cervix was significantly more common among EPTBs compared to LPTBs, $15.7 \%$ versus $1.8 \%$ respectively, within the SPTB group $(\mathrm{p}=0.007)$. Fe intake rates were significantly lower among the EPTBs in all three groups; but it was only the MIPTD group where anemia was significantly more common among the EPTBs compared to the LPTBs (31.6\% versus $13.6 \%$ respectively, $\mathrm{p}=0.022)$. Infection with the rate of $24.8 \%$ and placental problems with the rate of 26.3\% were more commonly associated with EPTBs rather than LPTBs, in the PROMPTB and MIPTB groups ( $\mathrm{p}=0.008$ and $p=0.001$ respectively). Goiter and diabetes, classified as other maternal disorders, mostly affected the LPTBs within the MIPTB group rather than the EPTBs $(20.5 \%$ versus 6.1 respectively, $\mathrm{p}=0.015$ ).

\section{Discussion}

Analysis of antenatal factors associated with PTBs and especially with earlier PTBs, is an important step of preventive strategies of public health. Our study defined some local antenatal factors in EPTBs compared to those in LPTBs; moreover, analyzed the EPTB profile associated with spontaneous PTB (SPTB), medically indicated PTB (MIPTB) and PTB with premature rupture of the membranes (PROMPTB). EPBs from Northwestern Turkey admitted to neonatal intensive care unit during years 2002-2010 were mostly secondary to spontaneous preterm labor $(n=140), 15.7 \%$ of which were associated with short cervix, and PPROM ( $\mathrm{n}=165), 24.8$ of which were associated with infections. EPTBs in the MIPTB group $(n=114)$, on the other hand, were frequently associated with hypertension in pregnancy $(66.7 \%)$.

The incidence of live-EPTBs (at 26/7-326/7 weeks) among all live births was 1.4\% (419/29841) in Northwestern Turkey during the study period. We could not reach the real numbers of live-LPBs (at 330/7-36 $66^{6 / 7}$ weeks), because some of these babies have received care in secondary NICUs, due to limited bed capacities and long hospital-stay time of the earlier born neonates at the tertiary NICU of Trakya University.

Total PTB rates are $22.3 \%$ in Bangladesh (where PTB rate at $28-31$ weeks is $2.9 \%), 12-13 \%$ in USA, 5-9\% in Europe, $5.1 \%$ in Iran, $12.6 \%$ in Africa, and $9.8 \%$ in Asia $(1,2,3,4)$. PTB is a syndrome with several phenotypes and associated with dif- ferent neonatal outcomes. Spontaneous PTB constitutes 22\% of all PTBs (5). In our study, SPTB rate was 33.79\% among all PTBs included in the study between years 2002 and 2010 .

The ratio of adolescent mothers was higher among EPTBs compared to LPTBs both in the SPTB group (10\% vs 3.6\%) and in the PROMPTB group (9.7\% vs $5.1 \%$ ), but not in the MIPTB group (7\% vs $14.2 \%$ ). The association of young maternal age with early PTBs ( $<34$ weeks) is also supported by several studies (6-9). Because of unsatisfactory antenatal care among young single mothers, there is a high risk of preterm birth and adverse obstetric events $(3,11-14)$. In our study, $72.3 \%$ of the EPTBs and $68 \%$ of LPTBs have received regular antenatal care, not significantly different from each other. Maternal education is significantly correlated with the risk of PTB across several countries $(3,10)$. Our study also emphasized this significant effect of education on PTBs. Particularly in SPTB and PROMPTB groups, the rate of lower educational level ( $\leq 8$ years) among EPTBs (32.9\% and $40 \%$ respectively) compared to LPTBs, was found to be appreciably high in this study.

The Canadian study pointed out the higher incidence of PTBs among smokers (22.2\%) compared to nonsmokers (12.4\%) (15). The rate of cigarette smoking was rather high both among EPBs (18.6\%) and LPTBs (20.3\%) in our study population.

The mechanism how prior PTB increases the risk in subsequent pregnancies as well, has not been well understood $(2,4,14)$. However, the recurrence of SPTB and MIPTB around the same gestational weeks may also support a common pathway between the two (16).

Antenatal vaginal bleeding due to placental and/or cervical problems may be a risk factor for PTB $(4,14)$. According to our study, ultrasonographically diagnosed placental problems were relatively more common among EPTBs (11.7\%), particularly in the MIPTB group (26.3\%). Short cervix, supposed to be an important risk factor, was involved in $8.1 \%$ of EPTBs, particularly in those with spontaneous onset of labor (15.7\%).

The incidence of PTB was reported to be significantly low $(9.7 \%)$ in treated asymptomatic infections compared with untreated controls $(22.3 \%)$ (17). In one other study, PTB was shown to be triggered by urinary tract infection around 26th30 th gestational weeks (4). In contrary, no significant relationship between PTB and urinary tract infections was also proposed (18). But, our regional study supports that infections (such as, urinary tract infection and chorioamnionitis) play an important role in EPTBs $(16.7 \%)$, especially in those associated with PPROM (24.8\%).

The rate of iron intake was lower among EPTBs (57.5\%) in comparison with LPTBs (84.8\%) in our study. But anemia was particularly common among the EPTBs within the MIPTB group (31.6\%). Nevertheless, the relation between antenatal 
iron intake and PTB is not clear. Antenatal iron intake throughout the gestation had no beneficial effect of on PTBs according to a study from Bangladesh which might be explained with the multifaceted pathogenesis of PTB (3). Additionally, a systematic review also reported no significant reduction in PTB rate with iron intake (19). Nevertheless, iron intake during pregnancy is encouraged in all pregnancies in Turkey.

Both malnutrition and obesity may increase the risk of PTB and the pregnancies under a balanced diet experience less PTB $(3,14)$. Analysis of subgroups in one study has indicated that prepregnancy obesity lowered the risk of SPTB (20). Our study showed that the incidence of normal weight mothers was significantly higher among EPTBs in comparison with LPBs (30.5\% vs $19.0 \%$ respectively), but that of overweight mothers was significantly lower ( $57.5 \%$ vs $77.8 \%$ respectively).

Our study has some limitations in consequence of intricate nature of antenatal features of PTB in our small sized study population. Therefore, this necessitates to extend the study from 2010 until now, both to observe the changing profile of antenatal features of PTBs, and to strengthen the statistical power. On the other hand, to take LPTBs as controls and also to analyze the PTBs according to onset of labor are attracting features of this study.

In conclusion, the analysis of antenatal features of EPTBs and comparison with those of LPTBs may shed a light to local health policies. Increasing education may reduce the numbers of adolescent pregnancies and control pre-pregnancy and antenatal maternal problems that may have reflection in decreasing the incidence of EPTBs in Northwestern Turkey.

: We acknowledge A. Betül Acunaş, Chair, Professor of Pediatrics, School of Medicine, Trakya University, for fruitful discussion that helped in writing the manuscript.

We have no competing interests to declare.

\section{References}

1. Blencowe H, Cousens S, Chou D, Oestergaard M, Say L, Moller AB, et. al. Born too soon: the global epidemiology of 15 million preterm births. Preterm Birth Action Group. Reprod Health 2013;10(Suppl 1):S2.

2. Goldenberg RL, Culhane JF, Iams JD, Romero R. Epidemiology and causes of preterm birth. Lancet 2008; 371(9606):75-84.

3. Shah R, Mullany LC, Darmstadt GL, Mannan I, Rahman $\mathrm{SM}$, Talukder RR, et al. Incidence and risk factors of preterm birth in a rural Bangladeshi cohort. the ProjAHNMo Study Group in Bangladesh. BMC Pediatr 2014;14:112-132.

4. Alijahan R, Hazrati S, Mirzarahimi M, Pourfarzi F, Hadi PA. Prevalence and risk factors associated with preterm birth in Ardabil, Iran. Iran J Reprod Med 2014;12(1):47-56.

5. Barros FC, Papageorghiou AT, Victora CG, Noble JA,
Pang R, Iams J, et.al. International Fetal and Newborn Growth Consortium for the $21^{\text {st }}$ Century. The distribution of clinical phenotypes of preterm birth syndrome: implications for prevention. JAMA Pediatr 2015;169:(3):220-9.

6. Kang G, Lim JY, Kale AS, Lee LY. Adverse effects of young maternal age on neonatal outcomes. Singapore Med. J 2015;56(3):157-63.

7. Restrepo-Méndez MC, Lawlor DA, Horta BL, Matijasevich A, Santos IS, Menezes AM, et. al. The association of maternal age with birthweight and gestational age: a cross-cohort comparison. Paediatr Perinat Epidemiol 2015;29(1):31-40.

8. Fall CH, Sachdev HS, Osmond C, Restrepo-Mendez MC, Victoria C, Martorell R, et al. Association between maternal age at childbirth and child and adult outcomes in the offspring: a prospective study in five low-income and middle-income countries (COHORTS collaboration). Lancet Global Health 2015;3(7):e366-77.

9. Pergialiotis V, Vlachos DG, Gkioka E, Tsotra K, Papantoniou N, Vlachos GD.Teenage pregnancy antenatal and perinatal morbidity: Results from a tertiary center in Greece. J Obstet Gynaecol 2014;19(6):1-5.

10. Ruiz M, Goldblatt P, Morrison J, Kukla L, Švancara J, Riitta-Järvelin M, et al. Mother's education and the risk of preterm and small for gestational age birth: a DRIVERS meta-analysis of 12 European cohorts. J Epidemiol Community Health 2015;69(9):826-33.

11. Olusanya B, Ofovwe G. Predictors of preterm births and low birthweight in an inner-city hospital in Sub-Saharan Africa. Matern Child Health J 2010;14(6):978-86.

12. Abrams E, Milner DA Jr, Kwiek J, Mwapasa V, Kamwendo DD, Zeng D, et al. Risk factors and mechanisms of preterm delivery in Malawi. Am J Reprod Immunol 2004;52(2):174-83.

13. Orvos H, Hoffman I, Frank I, Katona M, Pal A,Kovacs L. The perinatal outcome of pregnancy without prenatal care: A retrospective study in Szeged, Hungary. Eur J Obstet Gynecol Reprod Biol 2002;100(2):171-3.

14. Zhang YP, Liu XH, Gao SH, Wang JM, Gu YS, et al. Risk Factors for Preterm Birth in Five Maternal and Child Health Hospitals in Beijing. PLoS One 2012;7(12): e52780.

15. Mei-Dan E, Walfisch A, Weisz B, Hallak M, Brown R, Shrim A. The unborn smoker: association between smoking during pregnancy and adverse perinatal outcomes. J Perinat Med 2015;43(5):553-8.

16. Ananth CV, Getahun D, Peltier MR, Salihu HM, Vintzileos AM. Recurrence of spontaneous versus medically indicated preterm birth. Am J Obstet Gynecol 2006;195(3):643-50.

17. Farr A, Kiss H, Hagmann M, Marschalek J, Husslein P, Petricevic L. Routine Use of an Antenatal Infection Screen-and-Treat Program to Prevent Preterm Birth: 
Long-Term Experience at a Tertiary Referral Center. Birth 2015;42 (2):173-80.

18. Chen YK, Chen SF, Li HC, Lin HC. No increased risk of adverse pregnancy outcomes in women with urinary tract infections: a nationwide population-based study. Acta Obstet Gynecol Scand 2010;89(7):882-8.

19. Pena-Rosas JP, FE V. Effects and safety of preventive oral iron or iron-folic acid supplementation for women during pregnancy. Cochrane Database Syst Rev 2009; (4): CD004736.

20. Parker MG, Ouyang F, Pearson C, Gillman MW, Belfort MB, Hong X, et.al. Prepregnancy body mass index and risk of preterm birth: association heterogeneity by preterm subgroups. BMC Pregnancy Childbirth 2014;14:153-63. 